\title{
Bypassing of a barrier by dissociated and superlattice dislocations
}

\section{Bhushan, Karihaloo}

\section{Published in:}

Journal of Applied Physics

Link to article, DOI:

$10.1063 / 1.322190$

Publication date:

1975

\section{Document Version}

Publisher's PDF, also known as Version of record

Link back to DTU Orbit

Citation (APA):

Bhushan, K. (1975). Bypassing of a barrier by dissociated and superlattice dislocations. Journal of Applied Physics, 46(12), 5092-5096. https://doi.org/10.1063/1.322190

\section{General rights}

Copyright and moral rights for the publications made accessible in the public portal are retained by the authors and/or other copyright owners and it is a condition of accessing publications that users recognise and abide by the legal requirements associated with these rights.

- Users may download and print one copy of any publication from the public portal for the purpose of private study or research.

- You may not further distribute the material or use it for any profit-making activity or commercial gain

- You may freely distribute the URL identifying the publication in the public portal

If you believe that this document breaches copyright please contact us providing details, and we will remove access to the work immediately and investigate your claim 


\title{
Bypassing of a barrier by dissociated and superlattice dislocations
}

\author{
B. L. Karihaloo \\ Department of Solid Mechanics, The Technical University of Denmark, 2800 Lyngby, Denmark \\ (Received 4 March 1975)
}

\begin{abstract}
Very simple procedures are used to calculate the upper and lower bounds for the applied stress required for the leading extended (superlattice) dislocation in a group of $n$ coplanar screw dislocations of like sign with Burgers vector $b$ to bypass a noncoplanar perfect screw dislocation with Burgers vector $m b(m \ll n)$. Since these bounds are very close to each other, especially for small $m$, the long-range flow stress can be estimated accurately. The critical spacing between the parallel slip planes at the moment of bypass is determined in a natural way by studying the stability of equilibrium of the leader in the mobile group. Particular attention is paid to the effect of the stacking-fault (the antiphase boundary) energy and the barrier strength $m$ on the bypass stress.
\end{abstract}

PACS numbers: $61.70 . \mathrm{M}, 62.20$.

\section{INTRODUCTION}

It is now generally recognized that the main effect of the stacking-fault energy (SFE) in single crystals of pure metals and alloys, 1 at least in those of facecentered cubic structure, is on the stress required to initiate thermally activated cross-slip of dislocations, this stress being higher for materials of low SFE (high alloying content). The thermal component of the flow stress $^{2}$ as well as the contribution from the elastic interaction between forest and glide dislocations (which is of short-range nature) have been shown ${ }^{3}$ to form the dominant part of the total flow stress in stage I of the work-hardening process. At the same time, it has been demonstrated experimentally ${ }^{3}$ that in stage III of the work-hardening process (at large strains), the longrange stresses of athermal nature form a predominant part of the total flow stress-in accordance with the theoretical predictions, ${ }^{4}$ which suggest that the longrange stresses result from dislocation pile ups. The theoretical pile-up models consisted of a group of perfect $^{4,5}$ or extended ${ }^{6}$ dislocations piling up against the leader in the group. In such models cross-slip is easier because of the very-short-range nature of the obstacle. On the other hand, the nature of the obstacle clearly plays an important role in the cross-slip process. Thus cross-slip would be more difficult if the barrier were a preexisting lock rather than the leader in the mobile group. Such barriers as Cottrell-Lomer locks have been observed in fcc metals and alloys, ${ }^{7,8}$ although they do not occur in such a dense cluster as to form very strong obstacles through overlapping. Such strong obstacles would be required to withstand the very high stress concentrations predicted by the Seeger ${ }^{9}$ model.

Another approach to the study of the work-hardening phenomenon in fcc metals and alloys has been to characterize the behavior of pairs of extended (superlattice) dislocations on parallel slip planes. ${ }^{10-13}$ In practice, however, dislocations have been known to be present in large groups. ${ }^{7,8}$ An extension of this latter approach to such groups of dislocations would involve an appreciable computational effort.

In the light of the above considerations it was thought clearly desirable to reconsider the pile-up models with a view to establishing a relationship between the longrange flow stress and SFE [antiphase boundary energy
(APB) in the case of ordered alloys] using simple procedures that retain the physical reality of the discrete pile-up models ${ }^{14-16}$ and the mathematical simplicity of the continuous dislocation models ${ }^{17}$ without their accompanying disadvantages. Accordingly, very simple physical reasoning is used to obtain upper and lower bounds for the critical bypass stress. The lower bound procedure uses a mixed continuous-discrete dislocation model, in which the leading extended (superlattice) dislocation in the propagating group is allowed to remain discrete while the remainder are smeared into a continuous distribution; this procedure has been successfully applied in many physical situations. ${ }^{18-21}$ The upper bound for the critical bypass stress is furnished by the superdislocation approach, ${ }^{6,19}$ in which all but the leading mobile dislocation are replaced by a superdislocation of appropriate Burgers vector. These two approaches give bounds that are very close to each other, especially for $m \leqslant 1$, where $m$ is the ratio of the strength of the barrier to that of the individual mobile dislocation. The superdislocation approach also allows us to determine from mathematical principles the critical spacing between the slip plane and the plane of the barrier at which bypass occurs. This is made possible by a study of the equilibrium of the leader in the propagating group; the critical spacing corresponds to the onset of instability of the equilibrium of the leader which is predicted by mathematical theorems, well known in the theory of stability of motion. ${ }^{20,22}$

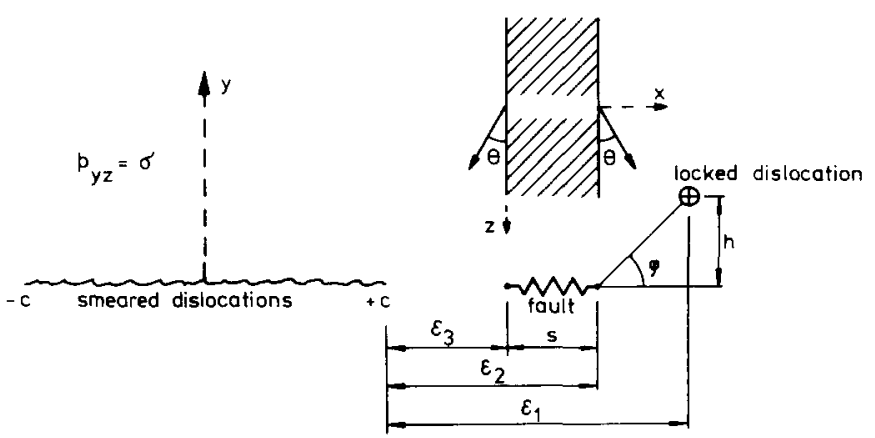

FIG. 1. Mixed continuous-discrete dissociated screw dislocation model for predicting the lower bound for the bypass stress. 


\section{LOWER BOUND FOR THE BYPASS STRESS}

The theoretical model, illustrated in Fig. 1, consists of $n$ positive (right-hand) extended screw dislocations (edges can be treated in an analogous manner), with Burgers vector $b$ parallel to the $z$ axis. Each dislocation is either dissociated into two energetically favorable partials that have Burgers vectors inclined at angle $\theta$ to the vector of the complete dislocation and that bound a ribbon of stacking fault whose energy is $\gamma$ or is of the superlattice type $\left(\theta=0^{\circ} ; \gamma\right.$, the antiphase boundary energy). In fcc metals and alloys perfect dislocation of the type $\frac{1}{2}[\overline{101}]$ can find it energetically favorable to split into two Shockley partials of the type $\frac{1}{2}\langle 112\rangle$, the angle $\theta$ being equal to $30^{\circ}$.

Additionally, there is a perfect screw dislocation with Burgers vector $m b$ locked along the plane $y=h$ and situated at $x=c+\epsilon_{1}$. The system is acted upon by an externally applied shear stress $p_{y z}=\sigma$. In accordance with the mixed continuous-discrete approach, the first extended mobile dislocation with its leading and trailing partials situated at $x=c+\epsilon_{2}, y=0$ and $x=c+\epsilon_{3}, y=0$, respectively, is allowed to remain discrete, while the remaining $(n-1)$ extended screws are smeared out into a continuous distribution within the interval $|x|<c$, it being implied that the latter possess no faults. Let the line joining the leading partial of the discrete extended dislocation with the barrier make an angle $\phi$ with the $x$ axis. The discrete free dislocation is in equilibrium at the position $\phi$ when the total forward stress acting on it due to the applied stress and the remaining free dislocations equals the repulsive stress due to the barrier. Now if the barrier is moved to the position $x$ $=c+\epsilon_{1}+h \tan \phi, y=0$ (Fig. 1) in line with the slip plane, the stress on the leading partial of the discrete free dislocations due to the barrier will remain unchanged while that on the remaining free dislocations decreases, as a result of which the mobile dislocations will move forward. By shifting the origin to the position originally occupied by the barrier, it is easy to show that the distance $A f(m, n) / \sigma$ to which the leading partial of the discrete mobile dislocation approaches the fixed dislocation in its new position is given by

$$
A f(m, n) / \sigma<h(\cot \phi+\tan \phi)
$$

or

$$
\sigma>f(m, n) A \sin (2 \phi) / 2 h .
$$

The lower bound of $\sigma$ may be assumed to occur at $\phi$ $=\frac{1}{4} \pi$. Although this assumption is not strictly correct, ${ }^{20}$ the values obtained in this way are, nevertheless, only slightly $(<1 \%)$ lower than the exact values obtained recently from elaborate mathematical calculations. ${ }^{20}$ This is also in close agreement with the results of Marcinkowski, Sadananda and Taunt, ${ }^{23}$ who found that in mode 1 bypass the applied stress attains a maximum at $\phi$ $\approx \frac{1}{4} \pi(y / x \approx 1$ in their Fig. 8). It therefore follows that for the leading free dislocation to bypass the barrier in the original model the lower bound for the critical stress is given by

$$
2 \sigma h / A>f(m, n),
$$

where $A f(m, n) / \sigma(A=\mu b / 2 \pi)$ is the distance to which the leading partial of the discrete free dislocation of a group of $n$ dislocations with Burgers vector $b$ approaches a fixed coplanar dislocation with Burgers vector $m b$ when the applied stress is $\sigma$. The function $f(m, n)$ has been evaluated recently ${ }^{21}$ and found to be

$f(m, n)=\left[\left(k_{2}-k_{1}\right) / 8 n\right]\left[1+\left(1+k_{1}\right)^{-1 / 2}+2 m\left(1+k_{2}\right)^{-1 / 2}\right]^{2}$,

where $k_{1}=\left(\epsilon_{2}-\epsilon_{3}\right) / \epsilon_{3}=s / \epsilon_{3}$ (s is the stacking-fault width or the separation between the components of the superlattice) and $k_{2}=\left(\epsilon_{1}-\epsilon_{3}\right) / \epsilon_{3}$ are determined from a system of two nonlinear algebraic equations whose coefficients are a function of the SFE, $\gamma$ (APB). ${ }^{21}$ The inequality (3) can thus be rewritten

$$
\frac{n \sigma h}{A}>\frac{n f(m, n)}{2} \text {. }
$$

The lower bound values for $\sigma$ as a function of the SFE (or APB) will be presented later together with the upper bound values obtained by the superdislocation approach, which is briefly described below.

\section{UPPER BOUND FOR THE BYPASS STRESS}

By using certain physical arguments similar to those used above it is easy to estimate ${ }^{18}$ an upper bound for $\sigma$. However, it is also known ${ }^{18,19}$ that exactly the same upper bound is furnished by the superdislocation approach in which all the extended free dislocations except the leader are replaced by a superdislocation with Burgers vector $(n-1) b$. As well as furnishing the upper bound for critical bypass stress this approach allows us to determine explicitly the corresponding value of the critical interplanar spacing $h$, thereby obviating the necessity of having to assume a critical spacing $a$ priori. ${ }^{23}$ The situation (when $n \gg m$ ) here is analogous to that of a single extended dislocation being subject to a stress which maintains the superdislocation in equilibrium.

Furthermore, since both the trailing and the leading components of the discrete extended screw dislocation are also in equilibrium and the distance between the superdislocation and the trailing component of the discrete extended (superlattice) dislocation is much larger than $\epsilon_{1}, \epsilon_{2}, \epsilon_{3}$, or $h$

$$
\begin{aligned}
& \frac{n \sigma}{(m+1) A}+\frac{2 \gamma}{b A}=\frac{1}{k_{1} \epsilon_{3}}\left[\frac{1}{2}\left(1-\frac{\tan ^{2} \theta}{1-\nu}\right)+\frac{m k_{1} k_{2} \epsilon_{3}^{2}}{k_{2}^{2} \epsilon_{3}^{2}+h^{2}}\right], \\
& \frac{n \sigma}{(m+1) A}-\frac{2 \gamma}{b A}=\frac{1}{k_{1} \epsilon_{3}}\left[\frac{m\left(k_{2}-k_{1}\right) k_{1} \epsilon_{3}^{2}}{\left(k_{2}-k_{1}\right)^{2} \epsilon_{3}^{2}+h^{2}}-\frac{1}{2}\left(1-\frac{\tan ^{2} \theta}{1-\nu}\right)\right] .
\end{aligned}
$$

For superlattice screw dislocations $\theta=0^{\circ}$. For extended (superlattice) dislocations of edge orientation it is necessary to set $A$ equal to $\mu b / 2 \pi(1-\nu)$ instead of $\mu b / 2 \pi$ and replace $1 /(1-\nu)$ by $1-\nu$ in Eqs. (6). In addition, the term containing $h$ will be altered.

Equilibrium equations (6) of the components of the discrete extended screw dislocation can be combined and written

$$
\begin{aligned}
(1- & \left.\frac{s}{s_{0}}\right)\left(1-\frac{\tan ^{2} \theta}{1-\nu}\right) \\
& =\frac{m k(1-k)}{(1-k)^{2}+\alpha^{2} k^{2} s_{0}^{2} / s^{2}}-\frac{m k}{1+\alpha^{2} k^{2} s_{0}^{2} / s^{2}},
\end{aligned}
$$




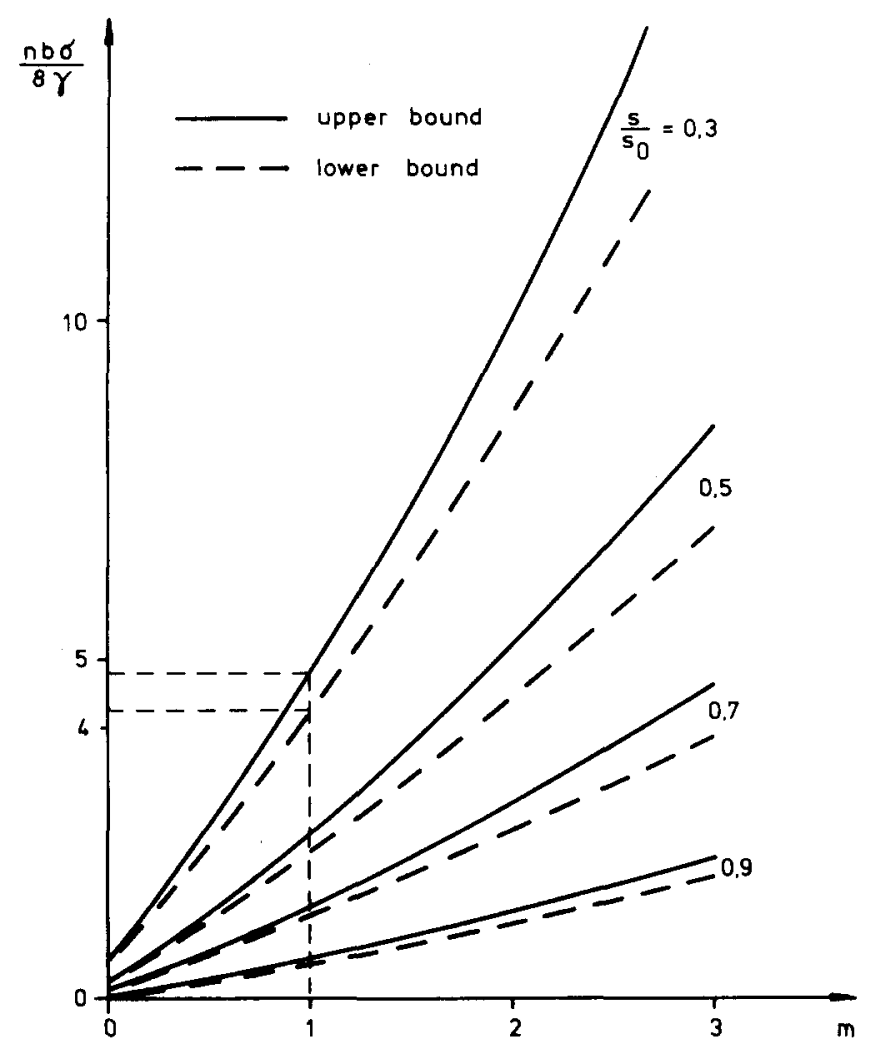

FIG. 2. Upper and lower bounds for the bypass stress $\sigma$ as a function of the degree of constriction $s / s_{0}$ and the strength of the barrier $m$ in the dissociated screw pile-up model $(\gamma, \mathrm{SFE})$. Also shown are the critical values of $s / s_{0}$ and $m$.

where $s=\epsilon_{2}-\epsilon_{3}=k_{1} \epsilon_{3}$ is the stacking-fault width, $k$ $=k_{1} / k_{2}$, and $\alpha=h / s_{0} . s_{0}$ is the equilibrium separation of the components in an unstressed lattice

$$
\begin{array}{rlrl}
s_{0} & =\frac{b A}{4 \gamma}\left(1-\frac{\tan ^{2} \theta}{1-\nu}\right) & & \text { (dissociated screws) } \\
& =\frac{b A}{4 \gamma} & \text { (superlattice screws) }
\end{array}
$$

Equation (7), which determines the critical interplanar spacing $h_{\mathrm{cri}}\left(\alpha_{\mathrm{\alpha ri}}\right)$ at which bypass occurs as a function of $s / s_{0}$ (degree of constriction of the dislocation ribbon), and the strength of the barrier $m$, can be rearranged as a fourth-order polynomial in $k$ :

$$
a_{0} k^{4}+a_{1} k^{3}+a_{2} k^{2}+a_{3} k+a_{4}=0,
$$

where

$$
\begin{aligned}
& a_{0}=\lambda_{1}^{2} \lambda_{2}+\lambda_{1} \lambda_{2}+m \lambda_{1}, \\
& a_{1}=m-2 \lambda_{1} \lambda_{2}, \\
& a_{2}=2 \lambda_{1} \lambda_{2}+\lambda_{2}-m, \\
& a_{9}=-2 \lambda_{2}, \\
& a_{4}=\lambda_{2},
\end{aligned}
$$

and

$$
\begin{aligned}
& \lambda_{1}=\frac{\alpha^{2} s_{0}^{2}}{s^{2}} \\
& \lambda_{2}=\left(1-\frac{s}{s_{0}}\right)\left(1-\frac{\tan ^{2} \theta}{1-\nu}\right) \quad \text { (dissociated screws) }
\end{aligned}
$$

$$
=1-\frac{s}{s_{0}}
$$

(superlattice screws).

From physical considerations $k<1$. In the terminology of the theory of stability of equilibrium, ${ }^{22} \mathrm{Eq}$. (9) will be called the "characteristic equation" of equilibrium of the discrete mobile dislocation. According to this theory, instability of equilibrium (and, by implication, onset of bypass) corresponds to its bifurcation, i. e., when two or more equilibrium paths are possible at the same value of external stress $\sigma$. In other words, for a given $s / s_{0}$ and $m$, the critical interplanar spacing is the one at which the characteristic equation of equilibrium has two positive real roots satisfying the physical requirement $k<1$. Certain mathematical theorems are invoked to arrive at the critical value $\alpha_{\mathrm{cr} 1}$ (Sec. IV). The stress corresponding to the onset of instability of equilibrium of the discrete dislocation may then be evaluated from one of the two Eqs. (6). This value sets an upper bound to the flow stress

$$
K \alpha_{\text {cri }}>n \sigma h / A \text {, }
$$

where

$$
\begin{aligned}
K= & (m+1) \frac{s_{0}}{s}\left[\frac{1}{2}\left(1-\frac{\tan ^{2} \theta}{1-\nu}\right)+\frac{m k}{1+\alpha_{\mathrm{cri}}^{2} k^{2} s_{0}^{2} / s^{2}}\right] \\
& -\frac{m+1}{2}\left(1-\frac{\tan ^{2} \theta}{1-\nu}\right) .
\end{aligned}
$$

Inequalities (4) and (12) specify the upper and lower bounds, respectively, for the flow stress

$$
\begin{aligned}
& \frac{n f(m, n)}{2}<\frac{n \sigma h}{A}<K \alpha_{c r i} \\
& \frac{n f(m, n)}{2 \alpha_{c r i}}<\frac{n \sigma b}{8 \gamma}<K .
\end{aligned}
$$$$
\text { or }
$$

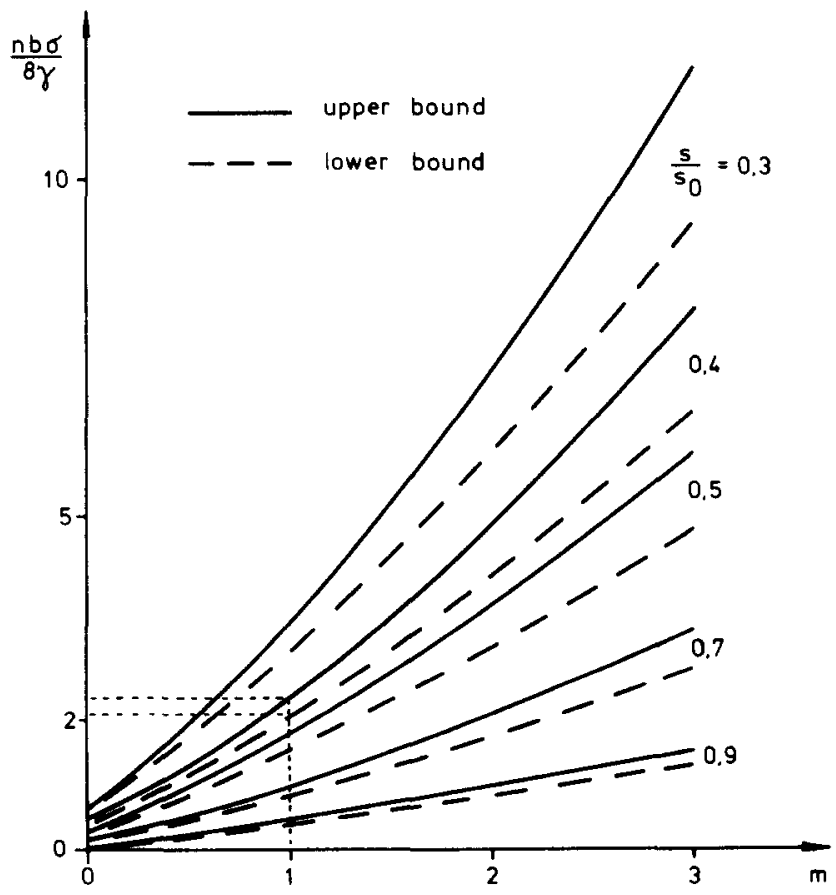

FIG. 3. Upper and lower bounds for the bypass stress $\sigma$ as a function of the degree of constriction $s / s_{0}$ and the strength of the barrier $m$ in the superlattice screw plle-up model 6, APB). Also shown are the critical values of $s / s_{0}$ and $m$. 
The upper and lower bounds for the long-range flow (bypass) stress as a function of the strength of the barrier and the degree of constriction are shown in Figs. 2 and 3, respectively, for extended and superlattice dislocations in fcc metals and alloys. $\nu$ has been assumed to be equal to $\frac{1}{3}$. It should be mentioned that the upper bound is taken as the mean of the two values corresponding to the two values of $k$ obtained as the roots of Eq. (9). Theoretically both these values of $k$ should give the same value of $\sigma$, but numerical errors are inevitable. The inaccuracy is, however, less than $\pm 2 \%$. It is clear that the bounds are very close to each other, especially for small $m$. Before discussing the results in the light of the experimental evidence available for fcc metals and alloys a brief analysis of the characteristic equation is presented.

\section{AN ANALYSIS OF THE CHARACTERISTIC EQUATION}

The critical value of $\alpha$ is the highest value at which the characteristic equation ( 9 ) has two positive real roots in the right-half plane $\operatorname{Re} k>0$; these two values of $k$ (which must at the same time satisfy the additional physical requirement $k<1$ ) correspond to the onset of instability of the equilibrium state of the leader in the mobile group. The applied stress required for the equilibrium state to bifurcate into two may then be evaluated from inequality (12). However, any analytical attempt to find $\alpha_{c r i}$ is, to say the least, a very difficult task. It is, therefore, expedient to invoke certain mathematical theorems about the roots of a polynomial. The one theorem which is eminently suited to our purpose is that due to Routh and Hurwitz ${ }^{22}$ or its simplified version due to Liénard and Chipart. ${ }^{22}$ The latter theorem establishes four sets of necessary and sufficient conditions and states that all the roots of a real polynomial have negative real parts if, and only if, any one of them is satisfied. We choose the one most convenient in our case, viz.,

$$
\begin{array}{ll}
a_{4}>0, & a_{2}>0, \quad a_{0}>0 ; \\
\Delta_{1}>0, & \Delta_{3}>0,
\end{array}
$$

where

$$
\Delta_{1}=a_{1} ; \quad \Delta_{3}=a_{1} a_{2} a_{3}-a_{1}^{2} a_{4}-a_{0} a_{3}^{2} .
$$

It is easy to show ${ }^{20}$ that subject to the condition that part of the coefficients be positive, violation of the condition $\Delta_{3}>0$ at a certain value of $\alpha$ amounts to the fourthorder polynomial having at least two roots with positive real parts. However, the roots with positive real parts could theoretically be imaginary. Thus inequalities (14) furnish us only with a starting value $\alpha_{0}$, which, as it happens, is almost equal to the critical value $\alpha_{\text {cri }}$ sought here. An examination of the coefficients $a_{k}$ $(k=0,1, \cdots, 4)\left[E q s_{\text {. }}(10)\right]$ shows that for all values of $s / s_{0}$ and $m$, the coefficients $a_{0}$ and $a_{4}$ are positive, while $a_{3}$ is negative. The coefficient most likely to change signs is $a_{1}$. A critical case would arise when $a_{1}=0$, i. e., $m=2 \lambda_{1} \lambda_{2}$, at which $\Delta_{3}<0$. Thus a starting value $\alpha_{0}$ is furnished by the relation $m=2 \lambda_{1} \lambda_{2}$, from which it follows that

$$
\alpha_{0}= \pm \frac{s}{s_{0}}\left(\frac{m}{1-s / s_{0}}\right)^{1 / 2} \quad \text { (dissociated screws), }
$$

$$
= \pm \frac{s}{s_{0}}\left(\frac{\frac{1}{2} m}{1-s / s_{0}}\right)^{1 / 2} \quad \text { (superlattice screws). }
$$

With this starting value of $\alpha$ the critical value $\alpha_{\text {cril }}$, at which the polynomial (9) had two positive real roots in the interval $0<k<1$, was computed numerically for various values of $s / s_{0}$ and $m$. Figure 4 shows the critical value of $\alpha$ as a function of $m$ for both the extended and the superlattice dislocation pile-up models in an fcc lattice.

The quotient of constriction $s / s_{0}$ has been chosen as 0.3 for the extended dislocation model on the following physical grounds. For most widely studied fcc metals the equilibrium separation of the Shockley partial dislocations (of the components forming a superlattice) $s_{0}$ is of the order of $15 b$ to $20 b$. For fcc alloys this separation increases with an increase in alloying content. Thus within the range of applicability of the continuum elasticity theory it is reasonable to allow the dislocation ribbon to shrink to about $5 b$ (of the order of the dislocation core radius), from which follows the assumed value of the constriction quotient. Furthermore, as is clear from Figs. 2 and 3, a lower value of this quotient would require the existence of very strong barriers ( $m$ very much greater than unity) to withstand the high stress concentrations. There is, however, no experimental evidence of the presence of such barriers in fcc metals and alloys. Only weak barriers $(m \leqslant 1)$ like LomerCottrell locks have been observed in such metals and alloys. ${ }^{7,8}$ Likewise, the interplanar distance $h_{\mathrm{cr} 1}$ $\left(=\alpha_{c r i} s_{0}\right)$ should not be so small as to render the continuum elasticity theory inapplicable. In general, it should not be less than about $1 b$ to $2 b .^{23}$ This latter condition restricts $s / s_{0}$ for superlattice models to about 0.4 . The values of $\alpha_{c r i}$ for this value of constriction quotient are also shown in Fig. 4. It is noteworthy that the values of $\alpha_{\text {cri }}$ for $s / s_{0}=0.3$ in the case of an extended dislocation model are practically equal to that of the superlattice model for $s / s_{0}=0.4$. The variation of the constriction quotient as a function of the critical interplanar spacing is shown in Fig. 5 for $m=1$. This particular value of the barrier strength is of special interest in discussing the flow process in fcc metals and alloys because it corresponds to a Lomer lock with

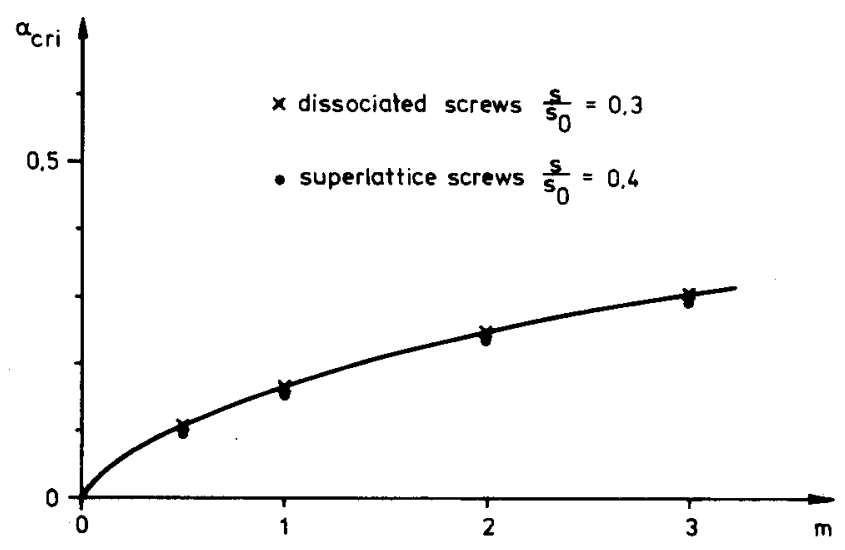

FIG. 4. Variation of the critical interplanar spacing $\alpha_{\mathrm{crt}}$ (corresponding to bypass) with the strength of the barrier $m$ for given degrees of constriction. 


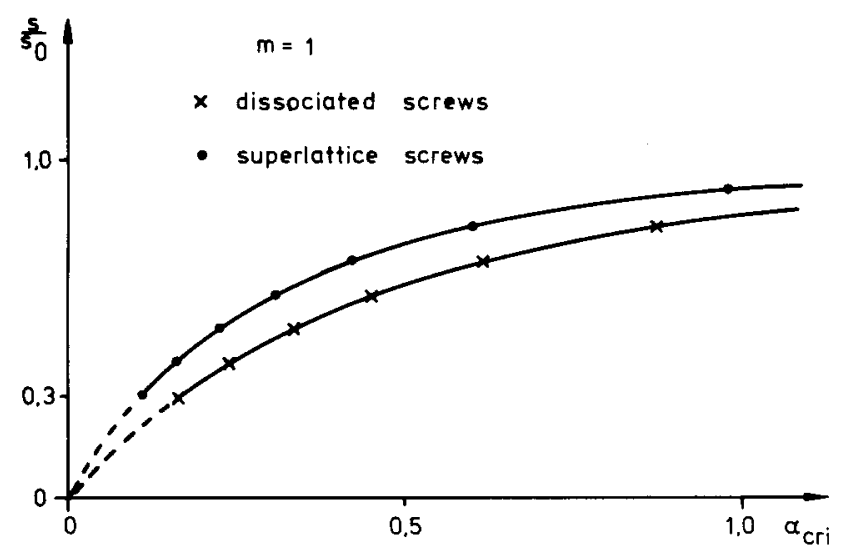

FIG. 5. Variation of the degree of constriction $s / s_{0}$ with the critical interplanar spacing $\alpha_{\text {cori }}$ for $m=1$.

Burgers vector $\frac{1}{2}[110]$. Strictly speaking, of course, the present analytical results will be in slight error in this case since the Burgers vector of the barrier is not parallel to that of the mobile dislocations, but the deviation should not be significant.

\section{DISCUSSION AND CONCLUSIONS}

On the physical grounds elucidated above it is quite reasonable to assume that the quotient of constriction should be about 0.3 and 0.4 , respectively, for the dissociated and superlattice pile-up models in a fcc lattice. This is also in agreement with the assumptions made elsewhere. ${ }^{23}$ Furthermore, experimental data ${ }^{7,8}$ pertaining to $\mathrm{Cu}$ and $\mathrm{Cu}-\mathrm{Al}$ alloys point to the presence of fairly large concentrations of Lomer-Cottrell locks in such alloys, although not in such clusters as to form strong barriers through overlapping. It is reasonable to assume that the strength of the barrier is less than unity $(m \leqslant 1)$. From Figs. 2 and 3 it then follows that $n b \sigma / \gamma$ should, on an average, have a value less than 18 and 36 for bypass to occur in superlattice and dissociated screw dislocation models, respectively. It is evident that $\sigma$ is linearly proportional to $\gamma$, a result also obtained elsewhere. ${ }^{13}$ The results obtained by the simple procedures used here can be directly interpreted in terms of the Seeger ${ }^{9}$ model for the onset of the third stage of work hardening in fcc metals and alloys. According to this model, the stress $\tau_{\text {III }}$ (corresponds to $\sigma$ in the present paper) at the onset of this stage is given by

$$
n \sigma b / \gamma=0.112 G b / \gamma-2 \text {. }
$$

For most widely studied fcc metals and alloys, like $\mathrm{Cu}$, $\mathrm{Ag}, \mathrm{Cu}-\mathrm{Al}$, and $\mathrm{Cu}-\mathrm{Ge}, \gamma / b G$ appears to be fairly independent of temperature up to about $200{ }^{\circ} \mathrm{C} .{ }^{24,25}$ Thus the results of the present work should be directly applicable in judging the validity or otherwise of the pile-up model predicting the onset of the stage III of work hardening. Thus, for $n \sigma b / \gamma<36(<18$ for the superlattice model), according to Eq. (16) $\gamma / b G$ should be greater than $3 \times 10^{-3}\left(6 \times 10^{-3}\right.$ for the superlattice model $)$. Now, the quantity $\gamma / b G \times 10^{3}$ for fcc metals $\mathrm{Cu}, \mathrm{Al}, \mathrm{Ag}$, and $\mathrm{Ni}$ is approximately equal to $15,34,7$, and 16 , respectively, ${ }^{26}$ whereas for their alloys $\mathrm{Cu}-10$ at. $\% \mathrm{Al}, \mathrm{Cu}-$ 10 at. $\% \mathrm{Zn}$, and $\mathrm{Ni}_{3} \mathrm{Mn},{ }^{23,27}$ the respective values are $1.5,1.4$, and 2 . The simple limit procedures used here allow us to conclude that the dissociated dislocation pile-up model with allowance for interplay between $s / s_{0}$ and $m$ can fairly accurately predict the onset of the third stage of work hardening for pure fcc metals with high SFE but not for their low stacking-fault alloys. The same conclusion is also valid for the superlattice dislocation model although it would seem to be in greater error for fcc alloys than the dissociated dislocation model. The superlattice model may, however, be more useful in ordered alloys. This is in close agreement with the results obtained by considering the bypass of a pair of superlattice dislocations on parallel slip planes. ${ }^{23}$ However, in contrast to the latter model ${ }^{23}$ the upper bound procedure used here allows us to determine the critical interplanar spacing from sound mathematical principles. It is worth mentioning that the lower bound procedure used here can easily be extended to cover the case of unlike dislocations ${ }^{13}$ whose mutual annihilation is a likely cause of the low work-hardening rate in stage III. However, the superdislocation approach can obviously not be applied. Finally, it should be emphasized that isotropic elasticity has been employed throughout the present study. It is quite likely that anisotropy and lattice friction could produce some perturbation, although it is hoped that the general nature of the conclusions would not be altered.

${ }^{1} \mathrm{H}$. Kronmuller, A. Seeger, and S. Mader, in Electron Microscopv and Strength of Crystals, edited by G. Thomas and J. Washburn (Interscience, New York, 1963), p. 665.

${ }^{2}$ Z.S. Basinski, Philos. Mag. 4, 771 (1959).

${ }^{3}$ C. K. L. Davies, V. Sagar, and R.N. Stevens, Acta Metall. 21,1343 (1973).

${ }^{4}$ P.M. Hazzledine and P. B. Hirsch, Philos. Mag. 15, 121 (1967).

${ }^{5}$ E. Smith, Proc. R. Soc. A 305, 387 (1968).

${ }^{6} \mathrm{E}$. Smith, Proc. R. Soc. A 315, 115 (1970).

${ }^{7}$ C.S. Pande and P.M. Hazzledine, Philos. Mag. 24, 1039 (1971).

${ }^{8}$ C.S. Pande and P.M. Hazzledine, Philos. Mag. 24, 1393 (1971).

${ }^{9}$ A. Seeger, R. Berner, and H. Wolf, Z. Phys. 155, 247 (1959).

${ }^{10}$ M.J. Marcinkowski and N.J. Olson, Philos. Mag. 18, 1111 (1969).

${ }^{11}$ K. Sadananda and M.J. Marcinkowski, J. Appl. Phys. 44, 4445 (1973).

${ }^{12}$ Shen-Ti Fong, M. J. Marcinkowski, and K. Sadananda, Acta Metall. 21, 799 (1973).

${ }^{13}$ M. J. Marcinkowski and K. Sadananda, J. Appl. Phys. 45, 2441 (1974).

${ }^{14}$ A. K. Head, Philos. Mag. 4, 295 (1959).

${ }^{15}$ J.D. Eshelby, F.C. Frank, and F.R.N. Nabarro, Philos. Mag. 42, 351 (1951).

${ }^{16}$ Y. T. Chou, J. Appl. Phys. 38, 2080 (1967).

${ }^{17}$ J.D. Eshelby, Philos. Mag. 40, 903 (1949).

${ }^{18} \mathrm{E}$. Smith, Philos. Mag. 22, 1161 (1970).

${ }^{19}$ E. Smith, Philos. Mag. 28, 293 (1973).

${ }^{20}$ B. L. Karihaloo, Proc. R. Soc. A (to be published).

${ }^{21}$ B. L. Karihaloo, Philos. Mag. (to be published).

${ }^{22}$ F. R. Gantmacher, Matrix Theory (Chelsea Publishing Co., New York, 1964), Vol. 2, p. 172 .

${ }^{23}$ M. J. Marcinkowski, K. Sadananda, and R. J. Taunt, Acta Metall. 22, 1405 (1974).

${ }^{24}$ P.C. J. Gallagher, Met. Trans. 1, 2429 (1970).

${ }^{25}$ T. Hasagawa, K. Asou, and A. S. Karashima, Met. Trans. 5 , 933 (1974).

${ }^{26} \mathrm{M}$. Ahlers, Met. Trans. 1, 2415 (1970).

${ }^{27}$ H. P. Karnthaler, P.M. Hazzledine, and M.S. Spring, Acta Metall. 20, 459 (1972). 